\title{
Differential release and deposition of S100A8/A9 proteins in inflamed upper airway tissue
}

\author{
Koen Van Crombruggen ${ }^{1}$, Thomas Vogl ${ }^{2}$, Claudina Pérez-Novo ${ }^{1}$, \\ Gabriele Holtappels ${ }^{1}$ and Claus Bachert ${ }^{1,3}$
}

Affiliations: ${ }^{1}$ Upper Airway Research Laboratory, Dept of Otorhinolaryngology, Ghent University Hospital, Ghent, Belgium. ${ }^{2}$ Institute of Immunology, University of Muenster, Muenster, Germany. ${ }^{3}$ Division of ENT Diseases, CLINTEC, Karolinska Institutet, Stockholm, Sweden.

Correspondence: Koen Van Crombruggen, Upper Airway Research Laboratory, Dept of Otorhinolaryngology, Ghent University Hospital, De Pintelaan 185, 9000 Ghent, Belgium. E-mail: koen.vancrombruggendugent.be

ABSTRACT Intracellular $\mathrm{Ca}^{2+}$-binding S100A8/A9 proteins gain novel functions when released during inflammation. The exact outcome of their extracellular function depends on the local tissue environment in which they are released; both anti-inflammatory and pro-inflammatory responses are described, modulating the immune system by binding Toll-like receptor (TLR)-4 or the receptor for advanced glycation end-products (RAGE). However, the contribution of the proteins in the pathophysiology of chronic rhinosinusitis (CRS) remains unclear.

Homomeric S100A8 and S100A9, and heteromeric S100A8/A9 proteins were evaluated in CRS with/ without nasal polyps (CRSw/sNP) and controls. Functional responses were assessed in polyp tissue stimulated with S100 proteins in the presence of TLR-4 and RAGE blocking antibodies.

S100A8, S100A9 and S100A8/A9 protein levels were significantly higher in CRSwNP patients, showing increased deposition on extracellular matrix (ECM) structures of CRSwNP tissue in contrast to CRSsNP and controls. In the presence of Staphylococcus aureus, S100A8/A9 is released from neutrophils and from the ECM. Extracellular S100A8 and S100A9 proteins induced increased levels of diverse inflammatory mediators via TLR-4 engagement.

The inflammatory/remodelling characteristics of CRSwNP specifically allow increased retention of S100A8, S100A9 and S100A8/A9 proteins in the ECM of CRSwNP tissue. Upon release, homodimeric proteins act as a local danger signal inducing inflammatory mediators, predominantly via TLR-4 activation.

@ERSpublications

Deposition of S100A8/A9 proteins on ECM of CRSwNP tissue released from neutrophils induces pro-inflammatory response http://ow.ly/Q2sV7

This article has supplementary material available from erj.ersjournals.com

Received: Jan 282015 | Accepted after revision: July 042015 | First published online: Oct 222015

Support statement: This work was supported by funding from The Research Foundation Flanders (FWO) (research project no. G.0641.10) to K. Van Crombruggen, a Special Research Fund BOF (no. 01J01113) to K. Van Crombruggen, the Interuniversity Attraction Poles Program-Belgian Science Policy (P7/30) to C. Bachert and the German Research Foundation (DFG) (CRC1009 B8) to T. Vogl. Funding information for this article has been deposited with FundRef.

Conflict of interest: None declared.

Copyright OERS 2016 


\section{Introduction}

Chronic rhinosinusitis with nasal polyps (CRSwNP) is a prevalent chronic inflammatory condition of the upper airways which is often accompanied by comorbid asthma, associated with a substantially impaired quality of life and reduced workplace productivity [1]. A diverse spectrum of factors including viral and bacterial insults $[2,3]$, together with epithelial barrier malfunctions and tissue remodelling [4-6] could be implicated in the aetiology of CRSwNP. However, despite massive research efforts to unveil the pathophysiology, the exact reason for a lack of resolution still remains poorly understood and appears to be multifactorial [7].

A novel set of molecules that could be implicated in sustaining the inflammatory reaction may be found within the host itself. Indeed, in addition to mediators of inflammation originating externally, some endogenous intracellular molecules from the host can be released into the extracellular space where they gain functions that are distinct from those during normal physiology, modulating inflammatory responses under pathological conditions. These molecules are collectively classified as damage-associated molecular patterns (DAMPs) [6]. S100A8 and S100A9 proteins possess all of the characteristics required to be included in the group of DAMPs $[8,9]$. S100A8 and S100A9 proteins are two of the 24 members of the multifunctional S100 family of cytoplasmic EF-hand helix-loop-helix $\mathrm{Ca}^{2+}$-binding proteins and are abundantly expressed in myeloid cells. Both S100A8 and S100A9 form noncovalently associated homo- and heterodimers $[10,11]$. The heterodimeric complex is the predominantly occurring form and considered relevant for normal physiology $[12,13]$. However, recent results have also shown that homodimers of S100A8 and S100A9 exhibit strong pro-inflammatory activities in various mouse models [14, 15].

Extracellularly, while both the homodimeric and heterodimeric proteins are described to possess chemotactic properties [13, 16, 17], the individual homodimeric S100A8 and S100A9, and the heterodimeric S100A8/A9 proteins often have distinct functions [18]. It has been shown that the antimicrobial activity of extracellular S100 proteins is only exhibited via divalent metal cation sequestration by the heterodimeric form S100A8/A9 [19, 20]. Conversely, the pro-inflammatory effects of these S100 proteins per se can be exclusively assigned to the homodimeric S100A8 and S100A9 forms [14]. S100A8 and S100A9 proteins are described to modulate the innate immune system by binding the receptor for advanced glycation end-products (RAGE) [21] and Toll-like receptor (TLR)-4 [14].

S100A8 and S100A9 proteins have been associated with human diseases such as rheumatoid arthritis, juvenile idiopathic arthritis, inflammatory bowel disease, cystic fibrosis and several auto-inflammatory diseases $[9,22]$. Although many functions have been identified and proposed for these proteins, their exact roles in various biological processes still remain to be defined. In particular, the implication of S100A8/A9 proteins and their potential mechanism of action in the pathophysiology of CRSwNP is not well investigated.

\section{Methods}

Patients

Patient clinical data can be found in table 1. More detailed information regarding the sampling of tissues, sera and nasal secretions can be found in the online supplementary material.

\section{Tissue homogenates}

Frozen tissue samples were homogenised by means of mechanical disruption as described in the online supplementary material.

S100A8, S100A9 and S100A8/A9 ELISA

S100A8, S100A9 and S100A8/A9 protein levels were measured in tissue homogenates, serum, nasal secretions, and tissue pellets/supernatants from tissue-cube experiments (see below) by means of commercially available ELISA kits (BMA Biomedicals, Augst, Switzerland). Tissue homogenates were prepared from nasal polyp tissue of CRSwNP patients, ethmoidal mucosal tissue from CRS without nasal polyps (CRSsNP) patients and from inferior turbinates of control subjects. Human ex-vivo tissue-cube fragment stimulation assays were performed on nasal polyps from CRSwNP patients. More detailed technical information regarding the ELISA kits is provided in the online supplementary material.

\section{S100A8, S100A9 and S100A8/A9 immunohistochemistry}

Paraffin and cryo-sections of human sinonasal tissues from controls (inferior turbinates), CRSwNP patients (inferior turbinates and nasal polyps) and CRSsNP patients (ethmoidal mucosa) were stained for S100A8, S100A9, S100A8/A9 and/or myeloperoxidase (MPO), as reported in detail in the online supplementary material. 


\section{TABLE 1 Patient clinical data and other characteristics}

\begin{tabular}{|c|c|c|c|}
\hline & Control & CRSwNP & p-value \\
\hline \multicolumn{4}{|l|}{ Clinical data } \\
\hline Subjects & 34 & 40 & \\
\hline Age years & $36(18-70)$ & $49.5(20-72)$ & $0.001^{\#}$ \\
\hline Males/females $\mathrm{n} / \mathrm{n}$ & $18 / 16$ & $24 / 16$ & 0.639 9 \\
\hline Smokers & 10 & 2 & $0.009^{\Uparrow}$ \\
\hline Atopy & 15 & 17 & $1^{\pi}$ \\
\hline Asthma & 6 & 20 & $0.007^{\pi}$ \\
\hline COPD & 0 & 2 & $0.496^{\text {9 }}$ \\
\hline AERD & 0 & 3 & $0.245^{\uparrow}$ \\
\hline Computed tomography score (Lund-Mackay) & $0(0-2)$ & $18(11-24)$ & $<0.0001^{\#}$ \\
\hline Polyp score (Davos) & $0(0-0)$ & $4(2-6)$ & $<0.0001^{\#}$ \\
\hline \multicolumn{4}{|l|}{ Tissue mediator levels } \\
\hline $\mathrm{IL}-5 \mathrm{pg} \mathrm{g}^{-1}$ tissue & BDL (BDL-79) & 83 (BDL-1229) & $<0.0001^{\#}$ \\
\hline IL-8 pg g $^{-1}$ tissue & $1772(284-11660)$ & $3901(380-33627)$ & $<0.001^{\#}$ \\
\hline ECP $\mathrm{ng} \mathrm{g}^{-1}$ tissue & $210(11-3894)$ & 7507 (458-49995) & $<0.0001^{\#}$ \\
\hline MPO $\mathrm{ng} \mathrm{g}^{-1}$ tissue & $834(350-5288)$ & 3085 (751-17469) & $0.003^{\#}$ \\
\hline Total IgE UA g ${ }^{-1}$ tissue & $17.5(1.9-559.9)$ & $251(11-3927)$ & $<0.0001^{\#}$ \\
\hline \multicolumn{4}{|l|}{$S A E-\lg E$} \\
\hline Positive subjects & $1^{+}$ & 15 & $0.0005^{\pi}$ \\
\hline $\mathrm{UA} \mathrm{g}^{-1}$ tissue & $0.15 \pm 0.14$ & $4.0 \pm 0.6$ & $0.0007^{\#}$ \\
\hline \multicolumn{4}{|l|}{ Patients per sample source } \\
\hline Tissue measurements & 31 & 40 & \\
\hline Serum measurements & 33 & 21 & \\
\hline Nasal secretion measurements & 15 & 15 & \\
\hline
\end{tabular}

Data are presented as $\mathrm{n}$, median (range) or mean \pm SEM, unless otherwise stated. CRSWNP: chronic rhinosinusitis with nasal polyps; COPD: chronic obstructive pulmonary disease; AERD: aspirin-exacerbated respiratory disease; IL: interleukin; ECP: eosinophil cationic protein; MPO: myeloperoxidase; SAE-IgE: specific IgE antibodies against Staphylococcus aureus enterotoxins; BDL: below detection limit. \#: MannWhitney U-test; " : Fisher's exact test; ${ }^{+}$: total $n=31$.

S100A8 and S100A9 gene expression analysis

RNA extraction, first-strand cDNA synthesis and real-time PCR amplification of S100A8 and S100A9 were performed on inferior turbinates of control subjects and on nasal polyps of CRSwNP patients as described in detail in the online supplementary material.

Human sinonasal ex vivo tissue-cube fragment stimulation assay

Fresh human tissues were processed as described previously [23]. For a detailed description on the specific methodology applied in this study, see the online supplementary material.

\section{Assessment of inflammatory mediators}

Tissue-cube supernatants and/or tissue homogenates were assayed for interleukin (IL)-1 $\beta$, IL-5, IL-6, IL-8, IL-10, IL-17 and tumour necrosis factor (TNF)- $\alpha$ levels by means of Luminex xMAP technology using a Fluorokine MAP Multiplex Human Cytokine Panel A kit (R\&D Systems, Minneapolis, MN, USA) on a Bio-Plex ${ }^{\text {TM }} 200$ Array Reader (Bio-Rad, Hercules, CA, USA). Eosinophilic cationic protein (ECP) levels were measured by UniCAP (Phadia, Uppsala, Sweden). A commercially available ELISA kit was used to measure the levels of transforming growth factor (TGF)- $\beta 1$ (R\&D Systems); acid was added during the ELISA procedure, resulting in physicochemical activation of latent TGF- $\beta$. Total TGF- $\beta$ concentrations are reported, including both active and latent forms.

\section{Assessment of cell death}

Lactate dehydrogenase (LDH) levels were measured by means of a commercial cytotoxicity detection kit (Roche, Basel, Switzerland) according to the manufacturer's guidelines, using an LDH standard from rabbit muscle (Roche).

\section{Statistical analysis}

The data obtained from the two patient groups were analysed by a nonparametric Mann-Whitney U-test, while the data derived from human ex vivo tissue-cube fragment stimulation assays were compared by 
means of a nonparametric Kruskal-Wallis test followed by Dunn's multiple comparison test. Categorical variables were analysed by means of Fisher's exact test. Correlation analysis was performed by the Spearman rank-order method yielding a Spearman rho $\left(r_{s}\right)$ coefficient. A p-value $\leqslant 0.05$ was considered to be statistically significant (GraphPad, San Diego, CA, USA).

\section{Results}

S100A8, S100A9 and S100A8/A9 protein levels in human sinonasal tissue

ELISA data show tissue levels of S100A8 (figure 1a), S100A9 (figure 1b) and S100A8/A9 proteins (figure 1c) to be significantly higher in nasal polyp tissue from CRSwNP patients compared with control inferior turbinate. Tissue S100A8/A9 levels highly correlated with tissue IL-8 and MPO levels in both controls (Spearman $\mathrm{r}_{\mathrm{s}}=0.8074, \mathrm{p}<0.0001$ and $\mathrm{r}_{\mathrm{s}}=0.941, \mathrm{p}<0.0001$, respectively; figure $2 \mathrm{a}$ and $\mathrm{b}$ ) and CRSwNP patients (Spearman $r_{s}=0.7778, p<0.0001$ and $r_{s}=0.875, p<0.0001$, respectively; figure $2 c$ and $d$ ). The correlations were absent for homodimeric forms (data not shown).

None of the other clinical parameters or inflammatory mediators reported in table 1 showed any relationship to the S100A8, S100A9 and S100A8/A9 protein levels.

\section{S100A8, S100A9 and S100A8/A9 immunohistochemistry}

Acetone-fixed cryo-sections showed no or very locally limited S100A9 immunoreactivity (clone IDCP1) in control inferior turbinates of nondiseased subjects (figure 3a), contrasting with a very pronounced S100A9 immunoreactivity around the basement membrane, blood vessels, and in the deeper ECM structures of inferior turbinate (figure $3 \mathrm{~b}$ ) and nasal polyp (figure 3c) tissue from CRSwNP patients (figure 3d).

In formalin-fixed paraffin sections from CRSwNP tissue, the same antibody against S100A9 stained monocytes (figure 3e) and neutrophils (figure 3f), which were more abundantly present in CRSwNP (figure $3 \mathrm{e}$ and $\mathrm{f}$ ) tissues compared with control tissue (figure $3 \mathrm{~g}$ ). Double staining with antibodies against MPO (figure 3h) and MPO together with S100A9 (figure 3i) in human polyp tissue confirmed that the major source of S100A8/A9 proteins is neutrophils.

In both paraffin and cryo-sections, similar results were obtained with the monoclonal antibody against S100A8 (clone S13.67) and with polyclonal antibodies against S100A8 and S100A9 (generated by T. Vogl) (data not shown).

\section{Gene expression for S100A8 and S100A9}

The relative levels of gene expression for S100A8 and S100A9 did not differ between inferior turbinates from controls and nasal polyps from CRSwNP patients (figure E3 in the online supplementary material).

\section{Functional effect of S100A8, S100A9 and S100A8/A9 proteins on inflammatory cytokine release ex vivo}

Stimulation of nasal polyp tissue fragments with 1 (data not shown) and $5 \mu \mathrm{g} \cdot \mathrm{mL}^{-1}$ recombinant S100A8 and S100A9 proteins resulted in a concentration-dependent increase in IL-1 $\beta$ ( $p<0.05$ and nonsignificant, respectively), TNF- $\alpha$ (both $\mathrm{p}<0.001)$, IL-10 (both $\mathrm{p}<0.001)$ and IL-17 $(\mathrm{p}<0.001$ and $\mathrm{p}<0.05$, respectively) levels in the tissue culture supernatants (figure $4 \mathrm{a}-\mathrm{d}$ ), while the levels of IL-5 and IL- 8 (figure $4 \mathrm{e}$ and $\mathrm{f}$ ), and IL-6, TGF- $\beta$ and ECP (figure E4 in the online supplementary material) were not affected. S100A8/A9 did not alter the release of any of the measured mediators (figure $4 \mathrm{a}-\mathrm{f}$ ).

The increases in IL-1 $\beta$, TNF- $\alpha$ and IL-10 levels induced by S100A8 and S100A9 were significantly reduced in the presence of anti-TLR-4 antibodies, while functional blocking of the RAGE receptor had no effect
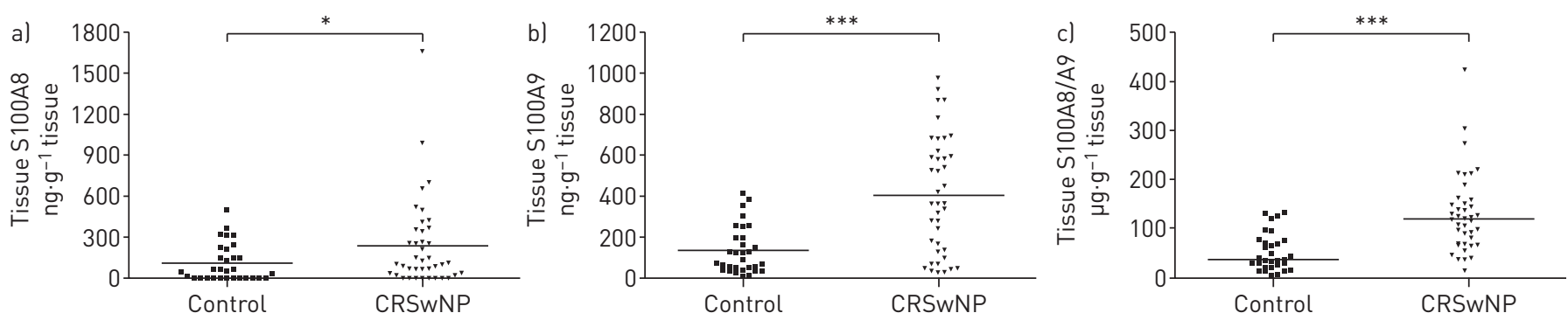

FIGURE 1 Sinonasal tissue levels of a) S100A8, b) S100A9 and c) S100A8/A9 proteins in controls and chronic rhinosinusitis with nasal polyps (CRSwNP) patients. Values presented as scatter plots showing the median as a line. ${ }^{*}: p<0.05$; ${ }^{* *}$ : $p<0.001$. The corresponding patient clinical data can be found in table 1 and table E1 (in the online supplementary material). 

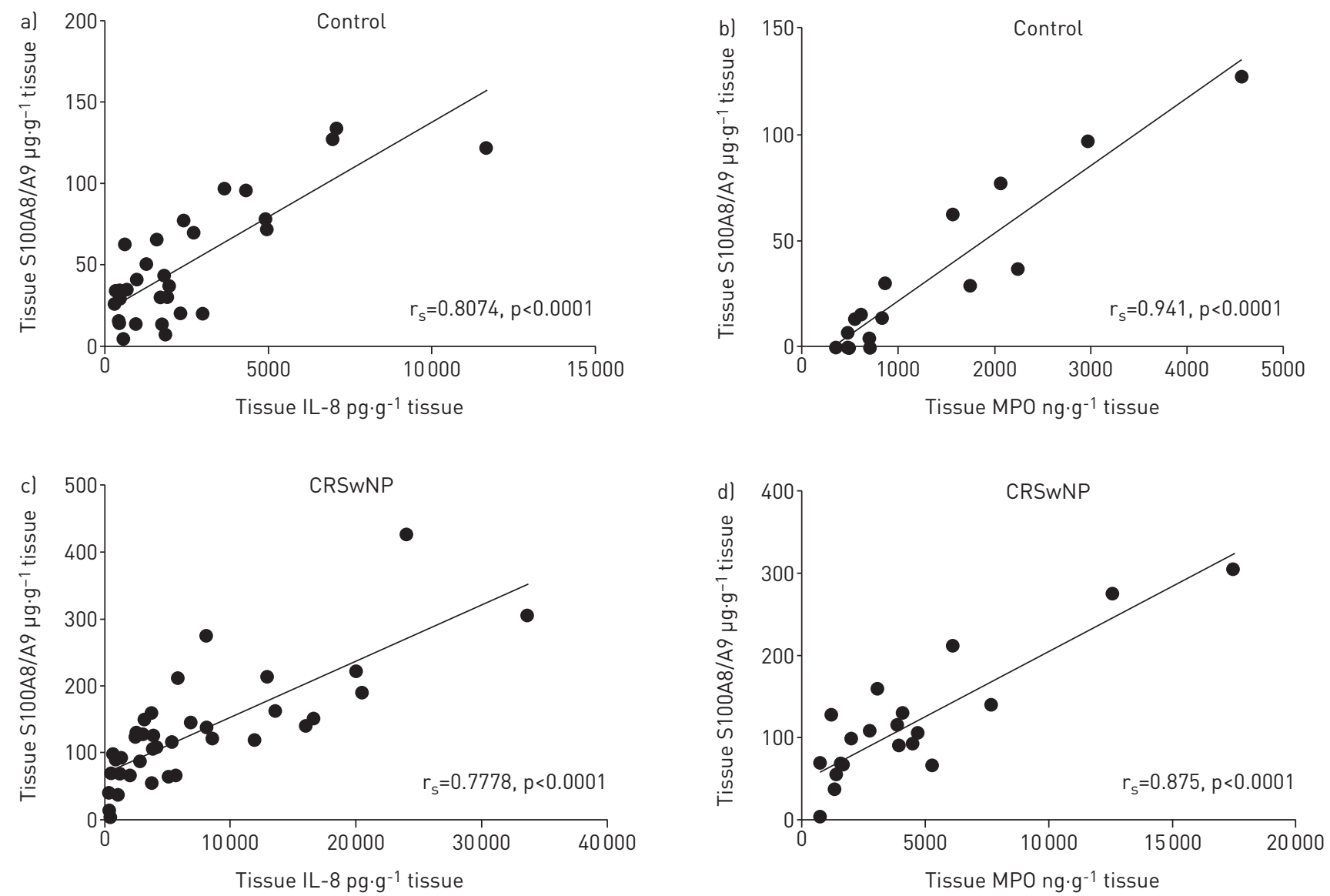

FIGURE 2 Correlation of tissue S100A8/A9 protein levels with tissue protein levels of interleukin (IL)-8 and myeloperoxidase (MP0) in a, b) controls (Spearman $r_{s}=0.8074, p<0.0001, n=30$ and $r_{s}=0.941, p<0.0001, n=17$, respectively) and $c$, d) chronic rhinosinusitis with nasal polyps (CRSwNP) patients (Spearman $r_{s}=0.7778, p<0.0001, n=38$ and $r_{s}=0.875, p<0.0001, n=22$, respectively).

(figure 5a-c). In contrast, the levels of IL-17 induced by S100A8 were significantly lowered in the presence of RAGE blocking antibodies, while TLR-4 blocking had no effect. Neither RAGE nor TLR-4 blocking could affect the levels of IL-17 induced by S100A9 (figure 5d). Neither the isotype control for TLR-4 nor the isotype control for RAGE had any effect (data not shown).

\section{Effect of Staphylococcus aureus on the release of S100 proteins ex vivo}

Under baseline conditions, S100A8/A9 time-dependently increased in the tissue-cube supernatants of the human nasal polyp tissue-cube fragment stimulation assay measured between 1 and $6 \mathrm{~h}$, and stabilised after $6 \mathrm{~h}$, as assessed at $24 \mathrm{~h}\left(1 \mathrm{~h}, 1530 \pm 610 \mathrm{ng} \cdot \mathrm{mL}^{-1} ; 6 \mathrm{~h}, 2010 \pm 750 \mathrm{ng} \cdot \mathrm{mL}^{-1} ; 24 \mathrm{~h}, 2000 \pm 620 \mathrm{ng} \cdot \mathrm{mL}^{-1}\right)$. Neutralisation of the IL-8 bioactivity did not affect the magnitude of S100A8/A9 release assessed at $24 \mathrm{~h}$ compared with control conditions (1910 \pm 310 versus $\left.2110 \pm 350 \mathrm{ng} \cdot \mathrm{mL}^{-1} ; \mathrm{n}=5\right)$.

In the presence of $S$. aureus, but not heat-inactivated $S$. aureus, a clear-cut increase in heterodimeric S100A8/A9 levels was observed in the tissue-cube supernatants at $24 \mathrm{~h}$, while the levels within the tissue-cube pellets were significantly decreased (figure 6a). A similar release of S100A8/A9 proteins by $S$. aureus was observed from peripheral blood-derived granulocytes (figure E7 in the online supplementary material) and to a lesser extent from peripheral blood mononuclear cells $\left(0.222 \pm 0.037 \mu \mathrm{g} \cdot \mathrm{mL}^{-1}\right.$ at baseline versus $2.037 \pm 0.127 \mu \mathrm{g} \cdot \mathrm{mL}^{-1}$ in the presence of $10^{5} \mathrm{CFU}$ S. aureus; $\left.\mathrm{n}=5, \mathrm{p}<0.05\right)$. Homodimeric S100A9 proteins could not be measured in the supernatants of the conditions described above, but S100A9 was present in the tissue-cube pellets and was significantly reduced by $S$. aureus but not by heat-inactivated S. aureus (figure 6b). Homodimeric S100A8 could not be measured in all of the samples described above (data not shown). Cell death could be excluded in these experiments as levels of LDH in the assay supernatants were not increased in the presence of $S$. aureus compared with baseline conditions (figures E7 and E8 in the online supplementary material). S. aureus-derived staphylococcal enterotoxin B and lipoteichoic acid had no effect on the levels of S100A8/A9 (data not shown). 

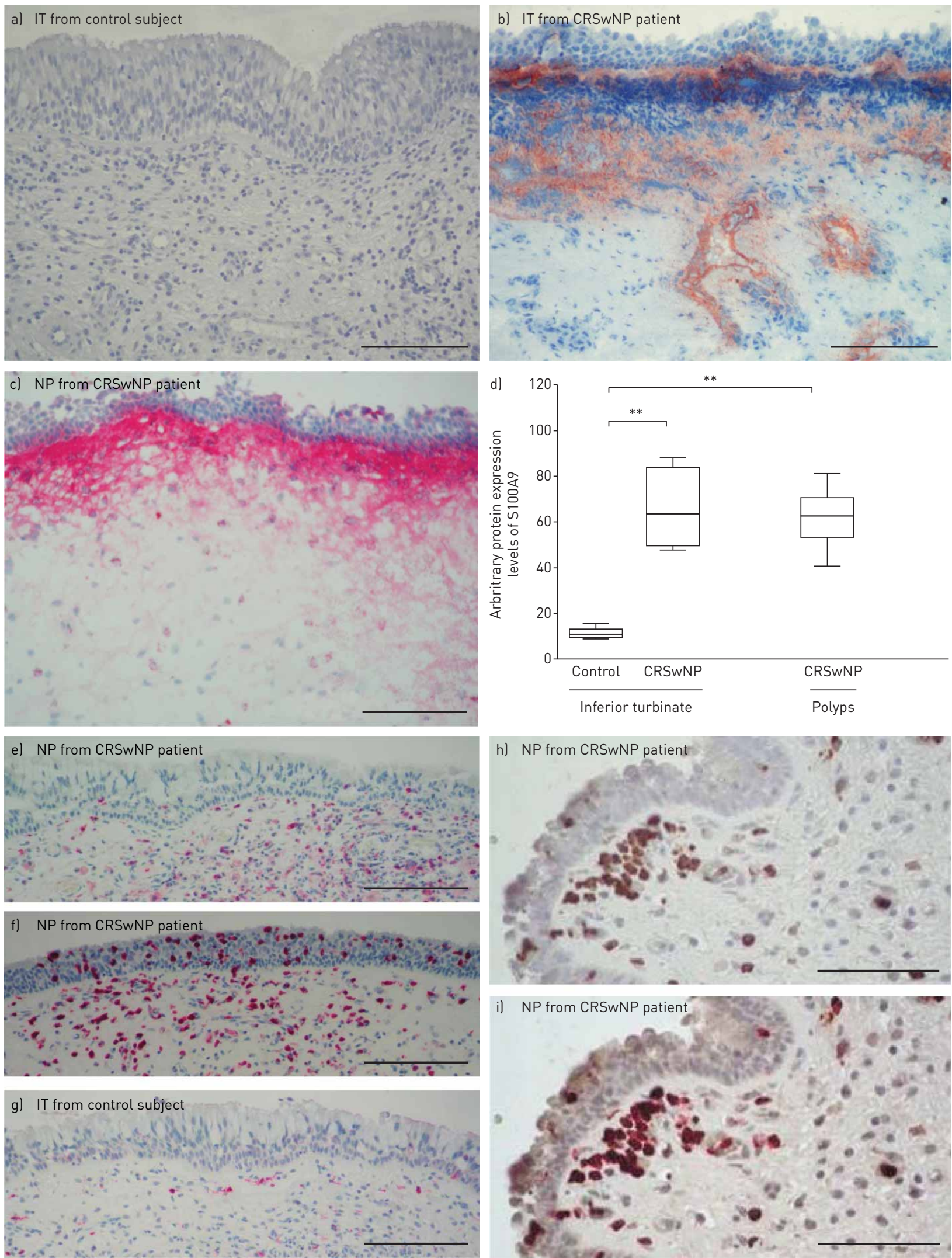

FIGURE 3 Immunostaining for S100A9 in inferior turbinate (IT) from a, g) control subjects, and b) inferior turbinate (IT) and c, e, f, h, i) nasal polyps (NP) from chronic rhinosinusitis with nasal polyps (CRSwNP). d) Semi-quantitative levels of immunostaining shown in panels a-c. ${ }^{* *}$ $p<0.01(n=6)$. Double staining with antibodies against h) myeloperoxidase (MPO) and i) MPO together with S100A9 confirmed that the major source of these S100 proteins is neutrophils. Staining in panels a-c was performed on acetone-fixed cryo-sections; staining in panels e-i was performed on formalin-fixed paraffin sections. Antibody clone IDCP1 was used for all stainings. Scale bars $=100 \mu \mathrm{m}$. 

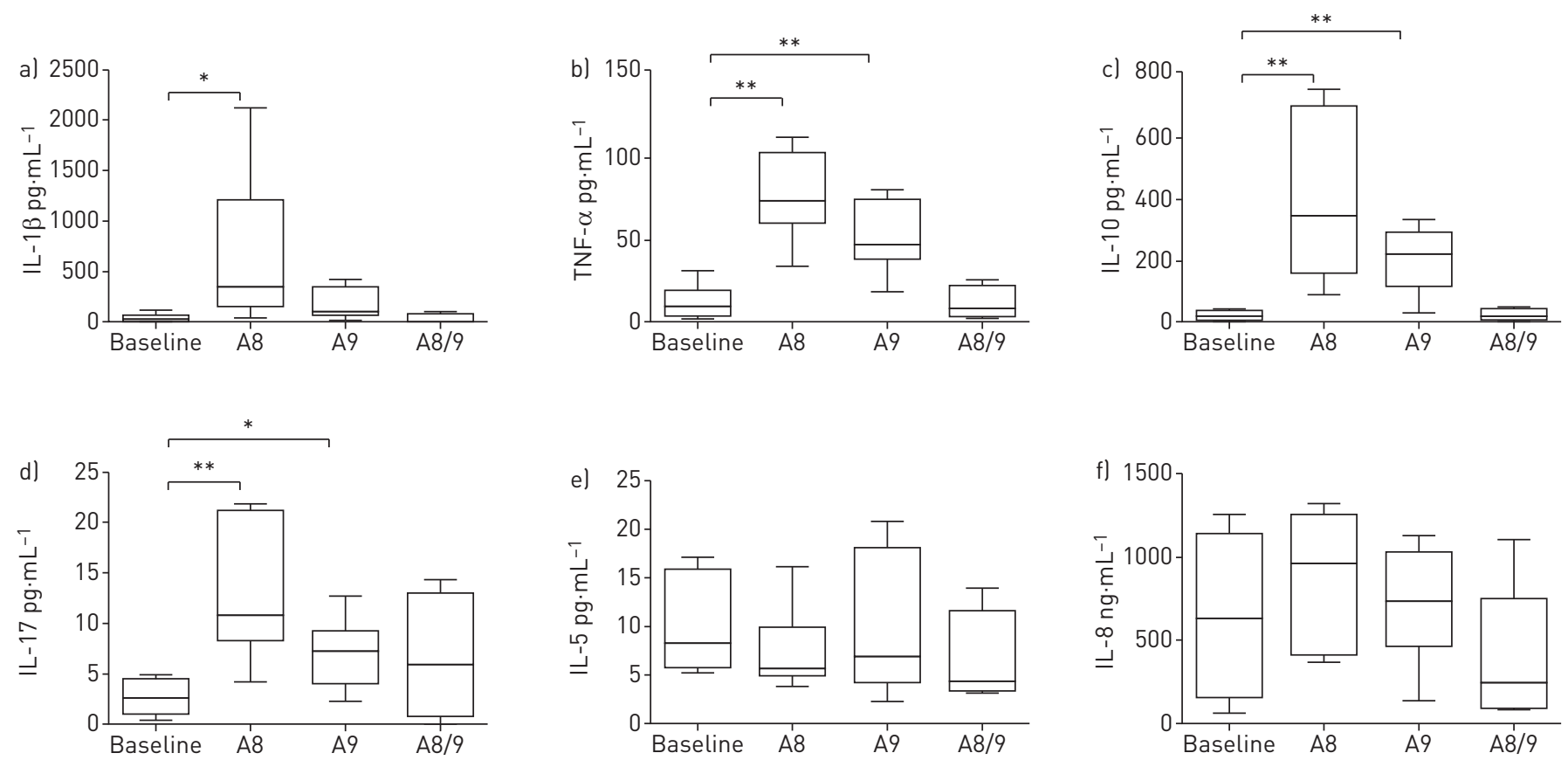

FIGURE 4 Effect of $5 \mu \mathrm{g} \cdot \mathrm{mL}^{-1}$ S100A8 (A8), S100A9 (A9) and S100A8/A9 (A8/9) proteins on release of a) interleukin (IL)-1 $\beta$, b) tumour necrosis factor (TNF)- $\alpha$, c) IL-10, d) IL-17, e) IL-5 and f) IL-8 proteins from human nasal polyp tissue in an ex vivo tissue assay. Data are presented as box-and-whisker plots showing minimum and maximum values, lower and upper quartiles, and the median $(n=6) .{ }^{*}: p<0.05 ;{ }^{* *}: p<0.01$.

The increased release of S100A8/A9 in the tissue-cube supernatants by $S$. aureus was significantly reduced by the tubulin polymerisation inhibitor demecolcine and the actin polymerisation inhibitor cytochalasin $\mathrm{B}$, while the de novo protein synthesis inhibitor cycloheximide and the endoplasmic reticulum/Golgi complex transport inhibitor brefeldin A had no effect (figure E9 in the online supplementary material).

\section{Discussion}

S100A8/A9 proteins comprise up to $45 \%$ of all cytosolic proteins in neutrophils and $~ 5 \%$ of all monocyte/ macrophage cytosolic proteins $[24,25]$. Indeed, a clear-cut infiltration of neutrophils that stained intensely positive for S100A8/A9 could be observed in paraffin sections of nasal polyp tissue. Moreover, we found S100A8 and S100A9 protein deposition in the extracellular matrix (ECM) of nasal polyps and inferior turbinates of CRSwNP patients, but not in CRSsNP patients or in control subjects. S100A8/A9 proteins were previously reported to interact with heparan sulfate proteoglycans [26] such as perlecan, agrin and collagen XVIII, which are present on ECM components, explaining our immunohistochemical stainings for S100A8 and S100A9. S100A8- and S100A9-immunoreactive vessels evidently do not synthesise these proteins themselves, but bind the S100A8/A9 proteins that appear to have been released by myeloid cells. It is suggested that when monocytes and neutrophils adhere to vascular endothelium, they release S100A8/A9 proteins onto the associated endothelium [27]. Our data, showing a correlation between tissue levels of IL-8 and S100A8/A9, while both proteins do not have a direct effect on their reciprocal release, can be brought in agreement with this mechanism of action. Indeed, IL-8 produced by activated epithelium or resident macrophages is known to be a strong chemoattractant for neutrophils which constitutively contain large amounts of S100A8/A9 proteins in their cytosol and consequently bring in these proteins in the CRSwNP tissue (figure 7). This influx of S100A8/A9 protein-containing cells and the observed protein deposition in the ECM can equally explain why the relative levels of gene expression for S100A8 and S100A9 do not differ between the tissue of controls and CRSwNP patients, whereas proteins levels do differ.

Although some S100A8/A9-containing myeloid cells can be found in nondiseased control tissues, even being abundantly present in CRSsNP, no deposition of S100A8/A9 proteins in the ECM structure could be observed in control and CRSsNP tissue, suggesting facilitated binding of the S100 proteins to the ECM under the specific pathology of CRSwNP. Indeed, tissue remodelling in CRSwNP [4] might change the ECM structure composition and thereby affect the ability of the $\mathrm{S} 100$ proteins to attach. In addition to increased retention in the ECM, alterations in the magnitude or release mechanisms of S100 proteins under inflammatory conditions can contribute equally to the increased levels of extracellular S100A8/A9 proteins. Indeed, our data show that $S$. aureus, a frequent coloniser of human upper airway mucosa with an increased prevalence in CRSwNP patients [4], can induce the release of S100A8/A9 from inflamed tissue of CRSwNP 

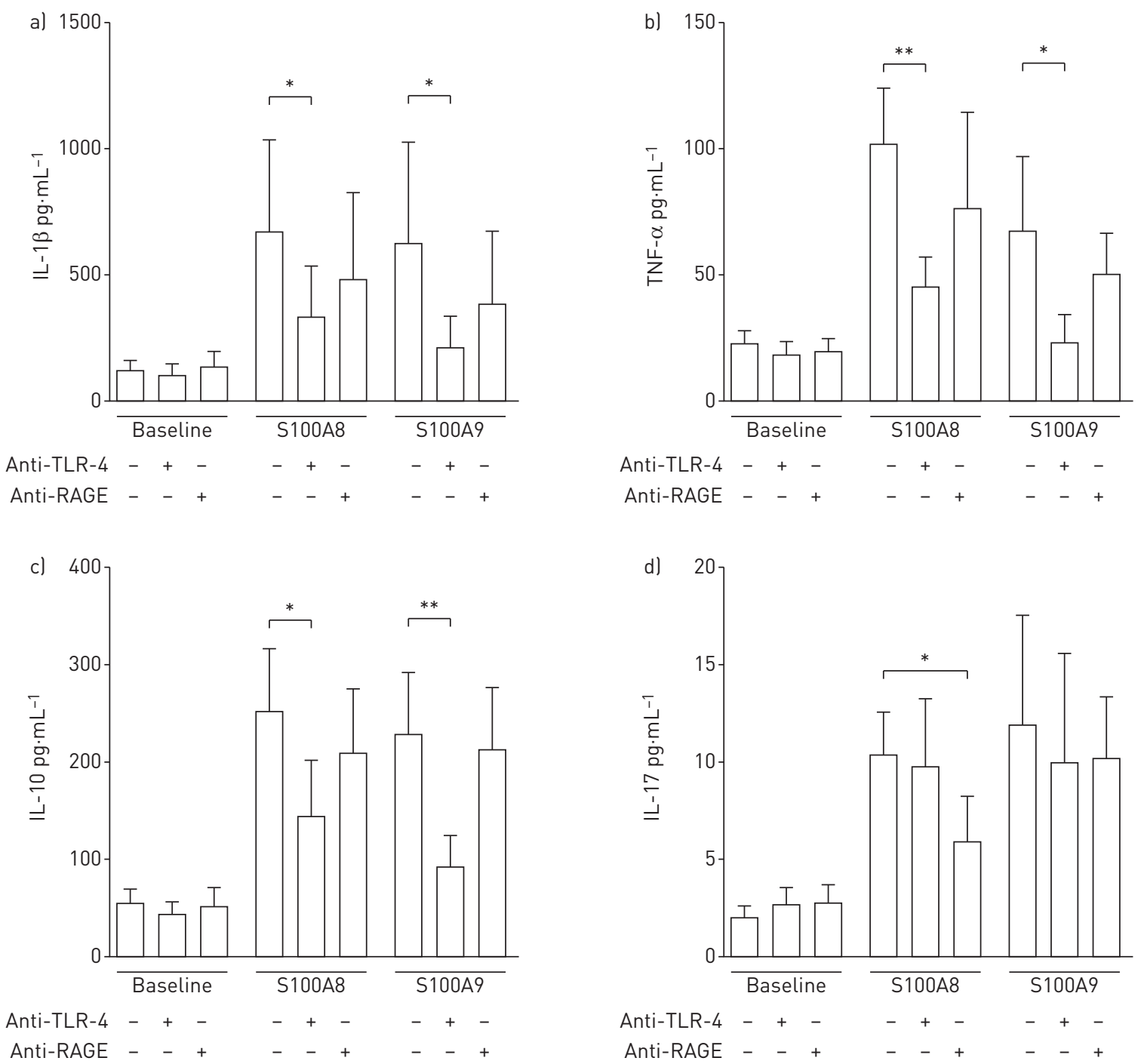

FIGURE 5 Effect of Toll-like receptor (TLR)-4 $\left(2 \mu \mathrm{g} \cdot \mathrm{mL}^{-1}\right)$ and the receptor for advanced glycation end-products (RAGE) (10 $\left.\mu \mathrm{g} \cdot \mathrm{mL}^{-1}\right)$ blocking antibodies on the responses induced by exogenous $5 \mu \mathrm{g} \cdot \mathrm{mL}^{-1}$ S100A8 and S100A9 proteins on the release of a) interleukin (IL)-1 $\beta$, b) tumour necrosis factor (TNF) $-\alpha, c)$ IL-10 and d) IL-17 proteins from human nasal polyp tissue in an ex vivo tissue assay. Data are presented as mean \pm SEM $(n=6){ }^{*}: p<0.05 ; * *: p<0.01$

patients and from peripheral blood-derived granulocytes and peripheral blood mononuclear cells. S. aureus is known to be implicated in the pathophysiology and severity of CRSwNP disease progression and the rate of developing comorbid asthma [28, 29]. Corroborated by the decreased levels of S100A8/A9 proteins measured in the CRSwNP tissue pellets, $S$. aureus induces a mechanism that liberates the cytosolic fraction of S100A8/A9 proteins from granulocytes. In view of the effect of brefeldin A, demecolcine and cytochalasin $\mathrm{B}$, the increased release of S100A8/A9 from neutrophils can be attributed to occur via a nonclassical secretory pathway during phagocytic cell migration and phagocytosis in which microtubule and actin-related pathways play important functions (figure 7). Phagocytosis-induced release of antimicrobial [20] heterodimeric S100A8/A9 proteins by neutrophils in CRSwNP can comprise a local environment $[19,30]$ optimised to combat S. aureus, a major contributor in the pathophysiology of CRSwNP [2].

In contrast to the direct antimicrobial effect of heterodimeric S100A8/A9 proteins [19, 20, 30], homodimeric S100A8 and S100A9 proteins also show the typical parameters of pro-inflammatory DAMPs [6]. In human CRSwNP tissue, we report that homodimeric S100A8 and S100A9 proteins induce a clear-cut increase in several inflammatory cytokines which are predominantly TLR-4-mediated [14], whereas RAGE [31] plays only a minor role (figure 7). In contrast, the heterodimeric S100A8/A9 protein per se yielded no inflammatory responses in our experiments. In addition to the release from the cytosol of myeloid cells into the extracellular environment, another prerequisite for the S100 proteins to induce the observed pro-inflammatory reactions thus relates to the availability of the homodimeric S100A8 and 

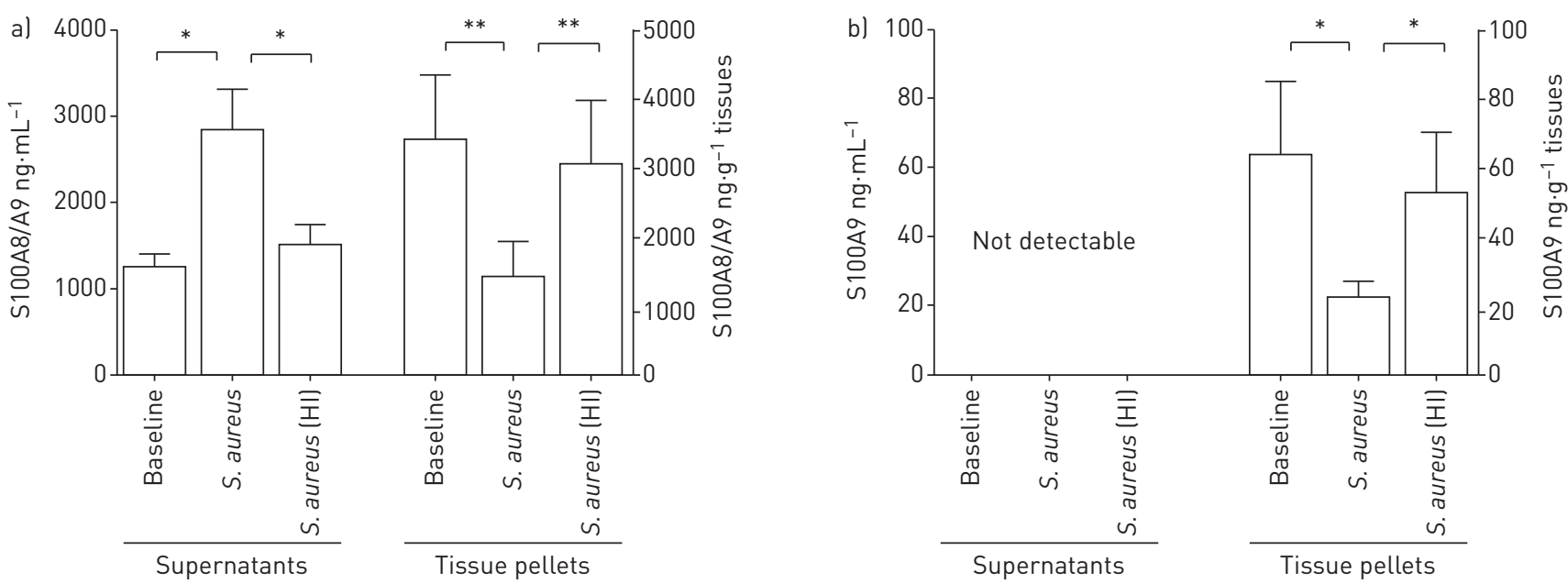

FIGURE 6 Effect of exogenously added $10^{5}$ CFU Staphylococcus aureus on the release of a) S100A8/A9 and b) S100A9 proteins from the human nasal polyp tissue into the supernatants in an ex vivo tissue assay. S100A8 was not detectable in the samples (data not shown). Data are presented as mean \pm SEM $(n=6-8)$. HI: heat-inactivated. *: $p<0.05 ;{ }^{* *}: p<0.01$.

\section{Epithelial barrier defence function}

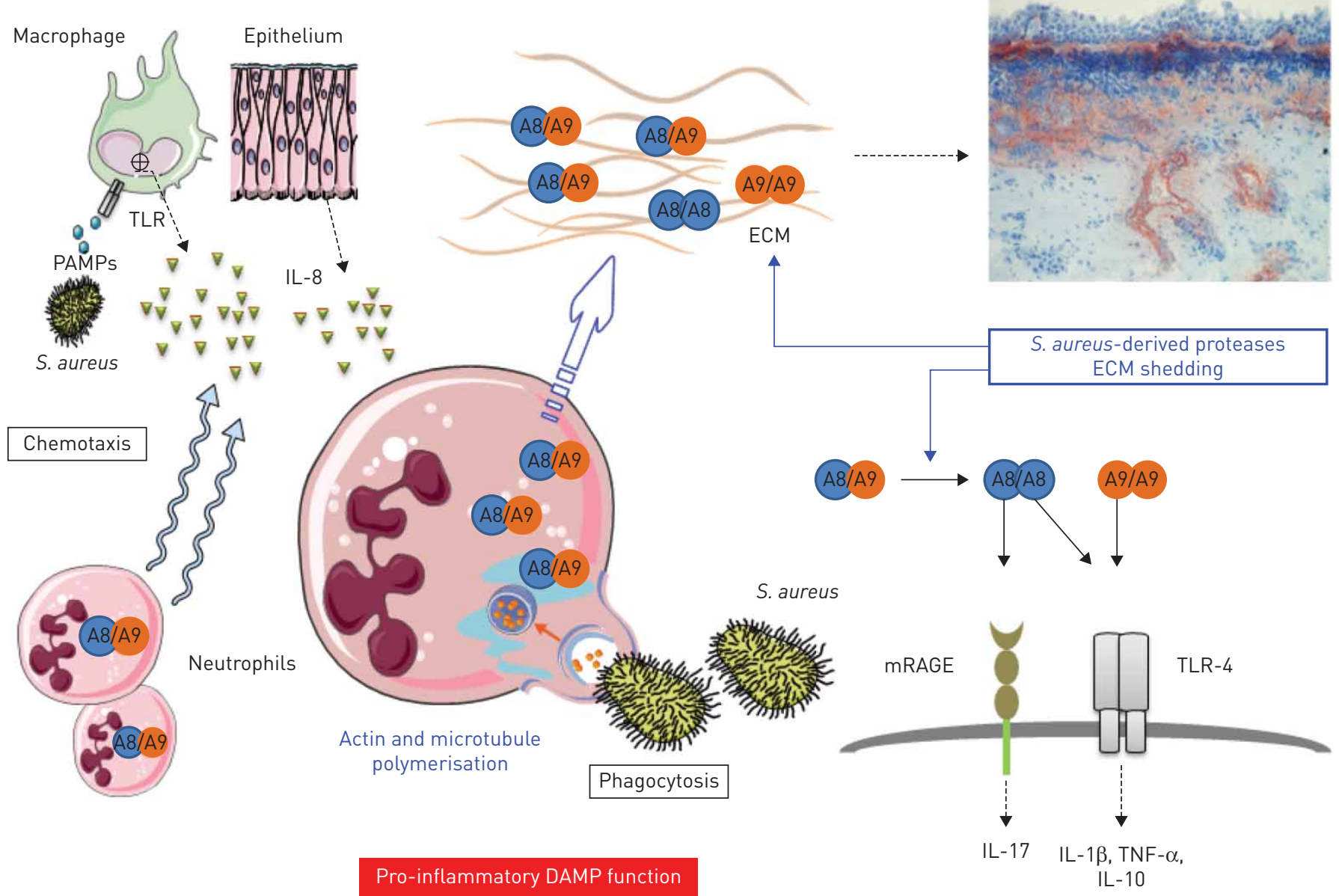

FIGURE 7 Proposed mechanism of action of $\mathrm{S} 100$ proteins contributing to the pathophysiology of chronic rhinosinusitis with nasal polyps as described in detail in the Discussion section. A8: S100A8; A9: S100A9; DAMP: damage-associated molecular pattern; ECM: extracellular matrix; IL; interleukin; mRAGE: membrane-bound receptor for advanced glycation end-products; PAMP: pathogen-associated molecular pattern; S. aureus: Staphylococcus aureus; TLR; Toll-like receptor; TNF: tumour necrosis factor. 
S100A9 proteins at the site of inflammation. Despite the fact that neutrophils exclusively release the heterodimeric S100A8/A9 form [32, 33], as evidenced from our ex vivo tissue and granulocyte model, both homodimeric S100A8 and S100A9 protein forms are present in the upper airway tissue, being significantly higher in CRSwNP patients. The mechanisms leading to the presence of homomeric proteins in the tissue of patients is consequently not due to a direct release from the neutrophils.

It can be speculated that ECM-associated heterodimeric S100A8/A9 proteins can be cleaved into the monomeric forms by a certain trigger that is present in the local tissue environment and subsequently be released from the matrix by the same or another trigger. When attached to the matrix, the monomers, which are normally not stable, might be protected from degradation by the local tissue environment and/ or form more stable homodimers via association with an unbound homologous monomer [34]. The binding of S100A8/A9 proteins to the ECM of CRSwNP tissue can be specifically attributed to the binding capacity of the S100A9 part of the dimer as it has been shown that S100A9 but not S100A8 binds to heparan sulfate proteoglycans [26]. When the ECM-bound S100A8/A9 protein dissociates under influence of a specific trigger (see below), the S100A9 proteins could remain intercalated in the ECM while the S100A8 monomers are released from the matrix, subsequently forming homodimers. Although the heterodimer is the preferential form, when one of the two monomers is present in excess over the other, homodimers are formed [32]. These homodimers are much more stable than the monomeric proteins, allowing them to induce their pro-inflammatory responses before being degraded, as they, in contrast to the heterodimeric S100A8/A9 proteins, are still prone to proteolytic degradation over time, which is especially true for S100A8 [35-37]. Indeed, also in the upper airways, the levels of S100A8 proteins from fresh homogenates were substantially lower when compared with the levels of S100A9 and S100A8/A9. When transferred to ex vivo culture conditions for $24 \mathrm{~h}$, endogenous homodimeric S100A8 proteins are not measurable anymore, whereas S100A8/A9 and S100A9 proteins were still clearly detectable in the tissue pellets. A second trigger might induce ectodomain shedding to subsequently induce the release of the ECM-associated homodimeric S100A9 proteins.

The increased prevalence of $S$. aureus in CRSwNP could bring into play such a set of triggers that might be able to split ECM-associated S100A8/A9 proteins into their monomeric forms because S. aureus is known to produce a diverse set of extracellular proteases, including serine proteases, cysteine proteases and metalloenzymes [38], which are often insensitive to human protease inhibitors, making them particularly aggressive. Moreover, S. aureus is also known to induce ectodomain shedding of host cell surface molecules to enhance their virulence [39], which might subsequently result in the release of the ECM-associated S100A9 proteins, explaining our ex vivo results showing S. aureus to reduce the levels of S100A9 proteins present in CRSwNP tissues (figure 7).

Our results suggest that homodimeric S100A9 proteins are protected from degradation during longer periods when attached to the ECM. Indeed, while S. aureus is able to reduce the levels of S100A9 proteins present in CRSwNP tissues, these proteins cannot be detected in the supernatants after $24 \mathrm{~h}$ ex vivo, indicating that they are indeed not $100 \%$ unsusceptible to degradation and are broken down over time when released from the ECM. A similar suggestion for protection against breakdown of the homodimeric S100A8 and S100A9 proteins by a local tissue environment could be made based on the phenotyping results of S100A9 knockout mice [37]. Similarly to what we propose for the human upper airway, this indicates that changes in local inflammatory status can affect protein stability and that in more peripherally located cells, this stability is jeopardised, probably by the lack of a protective environment. Hence the importance of studying these proteins under conditions resembling natural human physiology and pathology as close as possible, as we aim to do by means of our human sinonasal tissue model.

In conclusion, we report that S100A8, S100A9 and S100A8/A9 proteins are increased in sinonasal tissue of CRSwNP patients as a consequence of increased deposition on ECM structures in addition to the influx of S100A8/A9-containing inflammatory cells. Although neutrophils are more abundantly present in CRSsNP, this increased deposition in the tissue's ECM is only observed in CRSwNP, indicating that the specific inflammatory/remodelling milieu characteristic of CRSwNP allows the S100 proteins to attach. Heterodimeric S100A8/A9 proteins are released from neutrophils during phagocytosis of S. aureus, while the latter is able to release homodimeric S100A8 and S100A9 proteins from the ECM of CRSwNP tissue. Upon release, the homodimeric proteins show the potential to act as a danger signal by the release of inflammatory mediators, predominantly via TLR-4 activation in human CRSwNP tissue.

\section{References}

1 Bachert C, Zhang N, Holtappels G, et al. Presence of IL-5 protein and IgE antibodies to staphylococcal enterotoxins in nasal polyps is associated with comorbid asthma. J Allergy Clin Immunol 2010; 126: 962-968.

2 Van Zele T, Gevaert P, Watelet JB, et al. Staphylococcus aureus colonization and IgE antibody formation to enterotoxins is increased in nasal polyposis. J Allergy Clin Immunol 2004; 114: 981-983. 
Patou J, Gevaert P, Van Zele T, et al. Staphylococcus aureus enterotoxin B, protein A, and lipoteichoic acid stimulations in nasal polyps. J Allergy Clin Immunol 2008; 121: 110-115.

4 Van Bruaene N, Bachert C. Tissue remodeling in chronic rhinosinusitis. Curr Opin Allergy Clin Immunol 2011; 11: 8-11.

5 Zhang $\mathrm{N}$, Liu S, Lin $\mathrm{P}$, et al. Remodeling and inflammation in Chinese versus white patients with chronic rhinosinusitis. J Allergy Clin Immunol 2010; 125: 507-508.

6 Van Crombruggen K, Jacob F, Zhang N, et al. Damage-associated molecular patterns and their receptors in upper airway pathologies. Cell Mol Life Sci 2013; 70: 4307-4321.

7 Van Crombruggen K, Zhang N, Gevaert P, et al. Pathogenesis of chronic rhinosinusitis: inflammation. J Allergy Clin Immunol 2011; 128: 728-732.

8 Goyette J, Geczy CL. Inflammation-associated S100 proteins: new mechanisms that regulate function. Amino Acids 2011; 41: 821-842.

9 Ehrchen JM, Sunderkotter C, Foell D, et al. The endogenous Toll-like receptor 4 agonist S100A8/S100A9 (calprotectin) as innate amplifier of infection, autoimmunity, and cancer. J Leukoc Biol 2009; 86: 557-566.

10 Ishikawa K, Nakagawa A, Tanaka I, et al. The structure of human MRP8, a member of the S100 calcium-binding protein family, by MAD phasing at 1.9 A resolution. Acta Crystallogr D Biol Crystallogr 2000; 56: 559-566.

11 Itou H, Yao M, Fujita I, et al. The crystal structure of human MRP14 (S100A9), a Ca ${ }^{2+}$-dependent regulator protein in inflammatory process. J Mol Biol 2002; 316: 265-276.

12 Roth J, Burwinkel F, van den Bos C, et al. MRP8 and MRP14, S-100-like proteins associated with myeloid differentiation, are translocated to plasma membrane and intermediate filaments in a calcium-dependent manner. Blood 1993; 82: 1875-1883.

13 Vogl T, Ludwig S, Goebeler M, et al. MRP8 and MRP14 control microtubule reorganization during transendothelial migration of phagocytes. Blood 2004; 104: 4260-4268.

14 Vogl T, Tenbrock K, Ludwig S, et al. Mrp8 and Mrp14 are endogenous activators of Toll-like receptor 4, promoting lethal, endotoxin-induced shock. Nat Med 2007; 13: 1042-1049.

15 Loser K, Vogl T, Voskort M, et al. The Toll-like receptor 4 ligands Mrp8 and Mrp14 are crucial in the development of autoreactive $\mathrm{CD}^{+} \mathrm{T}$ cells. Nat Med 2010; 16: 713-717.

16 Ryckman C, Vandal K, Rouleau P, et al. Proinflammatory activities of S100: proteins S100A8, S100A9, and S100A8/A9 induce neutrophil chemotaxis and adhesion. J Immunol 2003; 170: 3233-3242.

17 Vandal K, Rouleau P, Boivin A, et al. Blockade of S100A8 and S100A9 suppresses neutrophil migration in response to lipopolysaccharide. J Immunol 2003; 171: 2602-2609.

18 Donato R, Cannon BR, Sorci G, et al. Functions of S100 proteins. Curr Mol Med 2013; 13: 24-57.

19 Kehl-Fie TE, Chitayat S, Hood MI, et al. Nutrient metal sequestration by calprotectin inhibits bacterial superoxide defense, enhancing neutrophil killing of Staphylococcus aureus. Cell Host Microbe 2011; 10: 158-164.

20 Sohnle PG, Hunter MJ, Hahn B, et al. Zinc-reversible antimicrobial activity of recombinant calprotectin (migration inhibitory factor-related proteins 8 and 14). J Infect Dis 2000; 182: 1272-1275.

21 Boyd JH, Kan B, Roberts H, et al. S100A8 and S100A9 mediate endotoxin-induced cardiomyocyte dysfunction via the receptor for advanced glycation end products. Circ Res 2008; 102: 1239-1246.

22 Chan JK, Roth J, Oppenheim JJ, et al. Alarmins: awaiting a clinical response. J Clin Invest 2012; 122: 2711-2719.

23 Van Crombruggen K, Holtappels G, De Ruyck N, et al. RAGE processing in chronic airway conditions: involvement of Staphylococcus aureus and ECP. J Allergy Clin Immunol 2012; 129: 1515-1521.

24 Hessian PA, Edgeworth J, Hogg N. MRP-8 and MRP-14, two abundant $\mathrm{Ca}^{2+}$-binding proteins of neutrophils and monocytes. J Leukoc Biol 1993; 53: 197-204.

25 Edgeworth J, Gorman M, Bennett R, et al. Identification of p8,14 as a highly abundant heterodimeric calcium binding protein complex of myeloid cells. J Biol Chem 1991; 266: 7706-7713.

26 Robinson MJ, Tessier P, Poulsom R, et al. The S100 family heterodimer, MRP-8/14, binds with high affinity to heparin and heparan sulfate glycosaminoglycans on endothelial cells. J Biol Chem 2002; 277: 3658-3665.

27 Hogg N, Allen C, Edgeworth J. Monoclonal antibody 5.5 reacts with p8,14, a myeloid molecule associated with some vascular endothelium. Eur J Immunol 1989; 19: 1053-1061.

28 Bachert C, Gevaert P, Howarth P, et al. IgE to Staphylococcus aureus enterotoxins in serum is related to severity of asthma. J Allergy Clin Immunol 2003; 111: 1131-1132.

29 Bachert C, Zhang N, Van ZT, et al. Staphylococcus aureus enterotoxins as immune stimulants in chronic rhinosinusitis. Clin Allergy Immunol 2007; 20: 163-175.

30 Corbin BD, Seeley EH, Raab A, et al. Metal chelation and inhibition of bacterial growth in tissue abscesses. Science 2008; 319: 962-965.

31 Sparvero LJ, Asafu-Adjei D, Kang R, et al. RAGE (Receptor for Advanced Glycation Endproducts), RAGE ligands, and their role in cancer and inflammation. J Transl Med 2009; 7: 17.

32 Hunter MJ, Chazin WJ. High level expression and dimer characterization of the S100 EF-hand proteins, migration inhibitory factor-related proteins 8 and 14. J Biol Chem 1998; 273: 12427-12435.

33 Vogl T, Leukert N, Barczyk K, et al. Biophysical characterization of S100A8 and S100A9 in the absence and presence of bivalent cations. Biochim Biophys Acta 2006; 1763: 1298-1306.

34 Riva M, He Z, Kallberg E, et al. Human S100A9 protein is stabilized by inflammatory stimuli via the formation of proteolytically-resistant homodimers. PLoS One 2013; 8: e61832.

35 Greenlee KJ, Corry DB, Engler DA, et al. Proteomic identification of in vivo substrates for matrix metalloproteinases 2 and 9 reveals a mechanism for resolution of inflammation. J Immunol 2006; 177: 7312-7321.

36 Nacken W, Kerkhoff C. The hetero-oligomeric complex of the S100A8/S100A9 protein is extremely protease resistant. FEBS Lett 2007; 581: 5127-5130.

37 Manitz MP, Horst B, Seeliger S, et al. Loss of S100A9 (MRP14) results in reduced interleukin-8-induced CD11b surface expression, a polarized microfilament system, and diminished responsiveness to chemoattractants in vitro. Mol Cell Biol 2003; 23: 1034-1043.

38 Dubin G. Extracellular proteases of Staphylococcus spp. Biol Chem 2002; 383: 1075-1086.

39 Park PW, Foster TJ, Nishi E, et al. Activation of syndecan-1 ectodomain shedding by Staphylococcus aureus alpha-toxin and beta-toxin. J Biol Chem 2004; 279: 251-258. 